\title{
Ceramide Synthases Are Attractive Drug Targets for Treating Metabolic Diseases
}

\author{
Suryaprakash Raichur* \\ Evotec International GmbH, Göttingen, Germany
}

\section{OPEN ACCESS}

Edited by:

Scott A. Summers,

The University of Utah, United States

Reviewed by:

Carsten Schmitz-Peiffer

Garvan Institute of Medical

Research, Australia

Yael Pewzner-Jung,

Weizmann Institute of Science, Israel

*Correspondence:

Suryaprakash Raichur

surya.prakash@evotec.com

Specialty section:

This article was submitted to

Diabetes: Molecular Mechanisms,

a section of the journal

Frontiers in Endocrinology

Received: 11 May 2020

Accepted: 22 June 2020

Published: 29 July 2020

Citation:

Raichur S (2020) Ceramide Synthases Are Attractive Drug Targets for

Treating Metabolic Diseases.

Front. Endocrinol. 11:483.

doi: 10.3389/fendo.2020.00483
Ceramide synthases (CerS) are central enzymes required for the de-novo synthesis of ceramides and other sphingolipids. They catalyze the addition of different acyl-chains to a sphingoid base, and thus account for much of the rich diversity in the sphingolipid family. Recent studies have demonstrated that the acyl-chain is an important determinant of ceramide function, such that a small subset of ceramides (e.g., those containing the $\mathrm{C} 16$ or $\mathrm{C} 18$ acyl-chain) alter metabolism by inhibiting insulin signaling or inducing mitochondrial fragmentation. Herein I discuss the therapeutic potential of targeting certain ceramide synthase isoforms for the treatment of obesity, insulin resistance, steatohepatitis, and other metabolic disorders.

Keywords: ceramides, sphingolipids, C16 ceramide, insulin resistance, obesity, NAFLD, NASH and mitochondrial dysfunction

\section{INTRODUCTION}

The increasing burden of metabolic diseases such as diabetes and heart disease is alarming, not only in developed countries, but throughout the world. Some prevalent metabolic disorders, such as non-alcoholic steatohepatitis (NASH), have no approved pharmacotherapies $(1,2)$. Unhealthy lifestyles fuel these pathologies, as combinations of sedentary lifestyles and poor dietary habits promote the delivery of excess saturated fatty acids and carbohydrates into non-adipose tissues such as liver and skeletal muscle, impairing their function $(3,4)$. Numerous studies reveal that the conversion of these excess fuels into sphingolipids such as ceramides is a critical event that leads to the cellular defects that accompany obesity $(5,6)$.

Clinical studies confirm that excess saturated fatty acid intake elevates levels of serum and tissue ceramides (7). Contrastingly, polyunsaturated fat intake reduces serum ceramides (7). In rodents, inhibiting ceramide biosynthesis using genetic and pharmacological approaches ameliorates atherosclerosis, hepatic steatosis, insulin resistance and obesity (8).

Two primary pathways produce ceramides in cells (Figure 1): (a) a de novo synthesis enzyme cascade that starts with the condensation of a saturated acyl-CoA (typically palmitoyl-CoA) and amino acid (typically serine) to produce the sphingoid backbone; and (b) a salvage pathway that involves the re-acylation of sphingosine. In both cases, ceramides (or dihydroceramides, in the case of the de novo synthesis pathway) are produced by ceramide synthases (CerS) through $\mathrm{N}$-acylation of the sphingoid base. Mammalian CerS exists in six isoforms (CerS1-6) with differing preferences for specific fatty acid chain lengths. CerS1 attaches $\mathrm{C} 18$ fatty acyl CoA to the sphingoid base; CerS2 attaches very long fatty acyl CoAs such as C22-C24; CerS3 attaches C26-C34 acyl CoA; CerS4 attaches C18-C20 fatty acyl CoA; and CerS5 and 6 have specificity for C14-C16 fatty acyl CoA. Thus, the CerS enzymes determine the acyl-chain composition of ceramides. CerS 


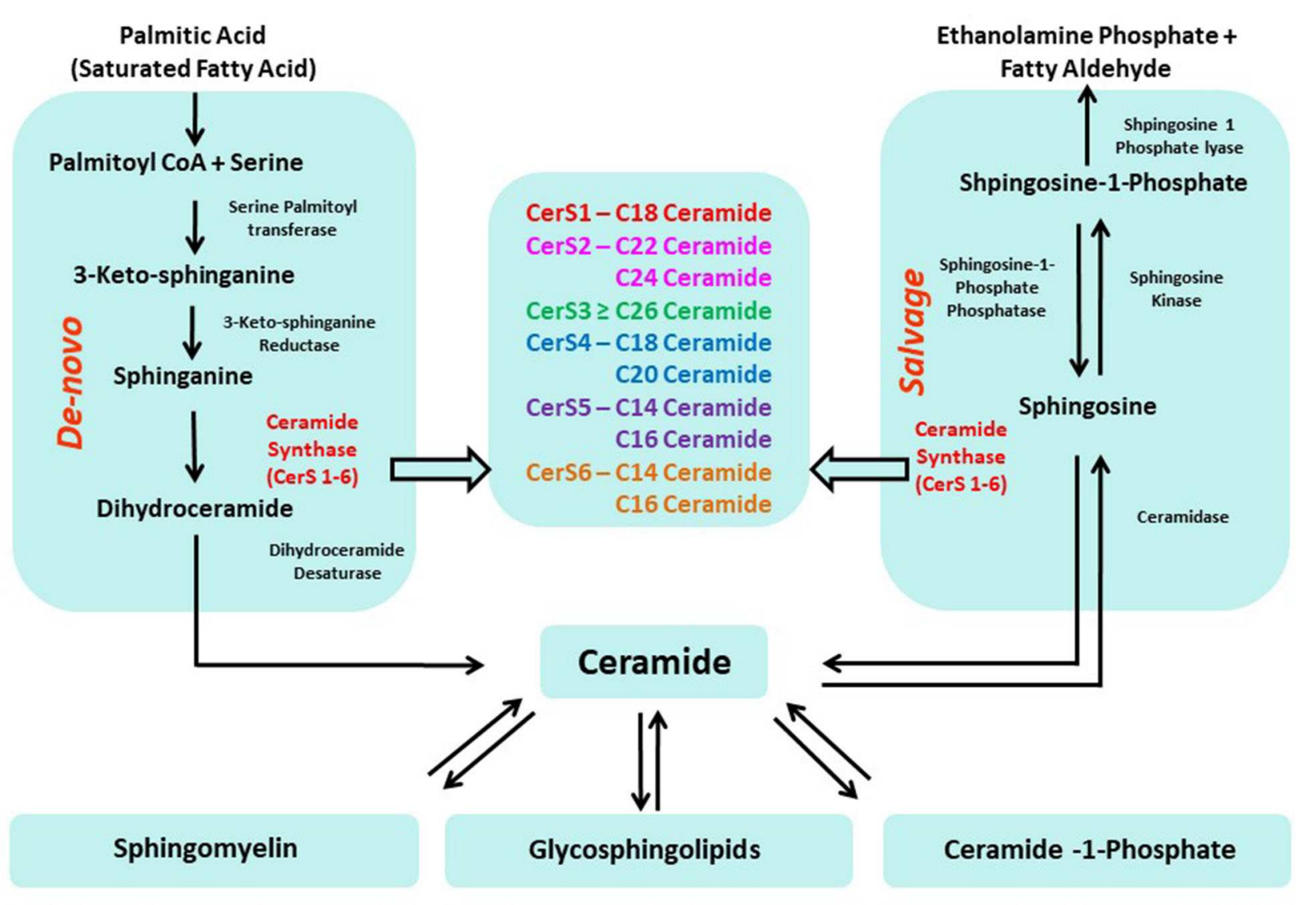

FIGURE 1 | Two primary pathways produce ceramides in cells: (a) de novo synthesis (b) re-acylation of sphingosine (salvage pathway).

expression, structure, localization, knock-out phenotypes, and association with other diseases have been reviewed comprehensively (9).

Ceramides can be further modified by the addition of different head groups, and the number of estimated bioactive sphingolipids range from 4,000 to 60,000 (10). The ceramides themselves have emerged as important signaling molecules that contribute to cellular stress responses (11). A primary mechanism through which ceramide promotes insulin resistance is by decreasing the activity of $\mathrm{Akt} / \mathrm{PKB}$, which is an essential facilitator of cellular glucose uptake. Ceramide blocks the activity of Akt/PKB by independent mechanisms: by enhancing Akt dephosphorylation via protein phosphatase 2A (PP2A) and by blocking the translocation of Akt via $\mathrm{PKCz}$ (12). Other ceramide actions have been identified that contribute to triglyceride production, mitochondrial dysfunction, and ultimately apoptosis (13).

Several genetic and pharmacological studies targeting de novo ceramide biosynthesis focused on the first enzyme in the pathway: serine palmitoyltransferase (SPT). Inhibiting this enzyme in rodents reduces global ceramide levels and ameliorates many forms of metabolic diseases (14). However, SPT is not a viable therapeutic target, owing to safety issues that result from the extreme diminution of all sphingolipids $(15,16)$. Therefore, therapeutic approaches focusing on a more limited subset of detrimental ceramides subspecies may represent a new strategy for therapeutic intervention. Fortunately, two recent reports demonstrated that the sphingolipid acylation patterns influences disease progression $(17,18)$, thus suggesting that the CerS enzymes might be viable targets.

\section{CLINICAL CORRELATIONS BETWEEN SPECIFIC CERAMIDES AND THE METABOLIC SYNDROME IN HUMANS}

Studies in clinical cohorts reveal striking relationships between serum or tissue ceramides and various measures of cardiometabolic disease. However, considerable variability exists regarding the precise sphingolipids that are most commonly elevated in a diseased individual. In the following paragraphs, I will focus on the larger studies that reveal specific roles for certain ceramides in diabetes and NASH.

\section{Insulin Resistance and Diabetes}

Insulin resistance, a condition typically defined as an inability of insulin to appropriately clear glucose from the bloodstream, 
is a risk factor for diabetes, heart disease, and NASH. Clinical observations reveal that circulating ceramides packaged in LDL (low-density lipoprotein) negatively associate with insulin sensitivity (19). The authors also demonstrated that infusion of LDL-containing ceramides into healthy mice attenuated whole body glucose clearance and increased levels of inflammatory markers (19), thus confirming that circulating lipids can be taken into tissues to alter metabolism. These authors also demonstrated that LDLs containing C16:0 and C24:0 ceramides reduced glucose uptake in cultured myotubes by inhibiting insulin signaling and decreasing translocation of the GLUT4 glucose transporter (19). In the Dallas Heart Study, consisting of 1,557 participants without type 2 diabetes assessed for metabolic biomarkers, fat depots and plasma ceramides over a period of 7 years, the saturated $\mathrm{C} 16$ and $\mathrm{C} 18$ ceramides correlated with insulin resistance (i.e., homeostatic model assessment of insulin resistance, HOMA-IR), total body fat and visceral adipose tissue (20). In contrast, healthier metabolic profiles were associated with longer-chain polyunsaturated fatty acid ceramides C24:2, C30:10, and C32:11 (20). Similarly, an evaluation of plasma sphingolipids in a large cohort of Chinese individuals in Singapore revealed that $\mathrm{C} 16, \mathrm{C} 18$, and $\mathrm{C} 20$ ceramides containing a d16:1 backbone correlated positively with body mass index (BMI) and HOMAIR (21). By comparison, hexosylceramides and ceramides with a d18:2 backbone negatively correlated with HOMA-IR and BMI (21). Off note, this study comprehensively assessed both the $\mathrm{d} 16: 1$ and $\mathrm{d} 18: 2$ backbone of sphingolipid species, whereas most of the studies generally focus on most abundant d18:2 backbone (21). Another interesting clinical study investigated ceramide subspecies and their ratios to determine the best predictors of diabetes (22). The authors identified C18/16 ratios as an independent marker for risk of diabetes incidence (22). Moreover, this ratio decreased in individuals following weight loss of $5 \%$ or more (22). A prospective study has revealed significant reductions in circulating very long chain ceramides (C20, C20:1, C22.1, C24, C26, C26:1) in type 1 diabetic patients that also associated with the development of nephropathy (23).

\section{Liver Disease}

A clinical study consisting of 406 patients with chronic viral hepatitis revealed that levels of sphingosine, sphinganine, and certain ceramides significantly associated with the severity of liver fibrosis in $\mathrm{HCV}$-infected patients (compared to $\mathrm{HBV}$-infected patients) (24). Moreover, Apostolopoulou et al. demonstrated that elevation of various sphingolipids species in the liver and serum of NASH patients compared to non-alcoholic fatty liver and control subjects (25). Specifically, in NASH, hepatic dihydroceramides (16:0, 22:0, and 24:1) and lactosylceramides increased significantly. Total serum dihydroceramides and hepaic dihydroceramides (22:0 and 24:1) increased significantly in NASH and strongly associated with whole body insulinresistance (25). Additional analysis shows that sphingolipid species correlated with hepatic oxidative stress and inflammation (25). In a prospective study consisting of 31 children diagnosed with non-alcoholic fatty liver disease (NAFLD), Wasilewska et al. demonstrated significant, positive correlation between total serum concentration of ceramides with insulin and also with HOMA-IR (26). Additionally, this study reveals that total ceramide concentration and specific (saturated fatty acyl) subspecies of ceramides such as C14, C16, C16:1, C18, and C18:1 were significantly higher in children with NAFLD compared to controls (26). Lastly, a randomized clinical trial conducted to determine the influence of dietary saturated and polyunsaturated fat on fatty liver development found that saturated fat markedly induces liver fat and serum ceramides (27). The effect was pronounced on C16 ceramides, whereas dietary polyunsaturated fat prevents liver fat accumulation and associated with reduced total ceramides (27).

\section{Bariatric Surgery}

The benefits of bariatric surgery on obesity and metabolic disease are well-established, including an almost immediate remission of type 2 diabetes and hyperlipidemia. In this context, surgically induced weight loss, which was associated with the improvement in insulin sensitivity and a decrease in proinflammatory cytokines, was shown to decrease plasma ceramide levels. Numerous serum ceramides, including the C16 subspecies, decreased in a time-dependent manner post-surgery (28). Subsequently, another study reveals a significant, timedependent reduction of serum $\mathrm{C} 22$ and C24 ceramides after laparoscopic sleeve gastrectomy (29).

Taken together, these clinical observations suggest that increases of saturated fatty acyl C16-18 ceramides, as well as several other sphingolipid species, are apparent in obese, fatty liver and insulin resistant individuals. The heterogeneity in species is a result of the dysregulated changes in ceramide biosynthesis in different organs. In this context, several mechanistic studies have found that the supply of saturated fat is sufficient to induce ceramides and cause metabolic dysfunction. For example, oversupply of saturated fatty acids by lipid infusion or diet promotes ceramide accumulation and activates inflammatory pathways, induces insulin resistance, impairs mitochondrial function, and stimulates endoplasmic stress and lipotoxicity. Blocking ceramide synthesis negates these saturated fatty acid actions $(6,8)$. Beyond saturated fatty acids, other factors, such as inflammatory signaling pathways, increase the rate of ceramide synthesis.

The above human lipodomic data (Table 1) also suggest that altered ratios of ceramide subspecies are associated with the comorbidities of diabetes and obesity. In this context, recent comprehensive sphingolipid analysis in rodent metabolic disease mouse models suggests that the ratio of long-chain ceramide species to very long-chain ceramide species in liver is a key marker of metabolic disease (32). Below I will discuss interventional studies in rodents suggest that accumulation of toxic species of ceramides such as C16 may play a role during progression of simple steatosis to NASH in humans.

\section{CERS ENZYMES AS THERAPEUTIC TARGETS}

The studies described above suggest that therapeutic interventions that reduce ceramides could have utility for 
TABLE 1 | Altered ceramide ratios in insulin resistance, diabetes, and fatty liver disease.

\begin{tabular}{|c|c|}
\hline \multicolumn{2}{|c|}{$\begin{array}{l}\text { Altered circulating/tissue long and very long chain ceramide ratios } \\
\text { associated with insulin resistance and diabetes }\end{array}$} \\
\hline \multirow[t]{2}{*}{ Neeland et al. (20) } & $\begin{array}{l}\text { Increased C16 and C18 ceramides in serum correlated } \\
\text { with insulin resistance, total body fat, and visceral fat } \\
\text { tissue }\end{array}$ \\
\hline & $\begin{array}{l}\text { Elevated levels of C24:2, C30:10, and C32:11 ceramides } \\
\text { in serum associated with healthier metabolic profiles }\end{array}$ \\
\hline Chew et al. (21) & $\begin{array}{l}\text { Increased C16, C18, and C20 ceramides in serum } \\
\text { correlated positively with body mass index (BMI) and } \\
\text { HOMA-IR }\end{array}$ \\
\hline Hilvo et al. (22) & $\begin{array}{l}\text { Increased C18 ceramide in serum shows the strongest } \\
\text { association with incident diabetes. } \\
\text { Study identifies C18/16 ratios as an independent marker } \\
\text { for risk of incidence of diabetes }\end{array}$ \\
\hline Klein et al. (23) & $\begin{array}{l}\text { Very long chain ceramides (C20, C20:1, C22.1, C24, } \\
\text { C26, and C26:1) are significantly reduced in serum of } \\
\text { type } 1 \text { diabetic }\end{array}$ \\
\hline Bergman et al. (30) & $\begin{array}{l}\text { Higher levels of C18 ceramide in skeletal muscle } \\
\text { association with insulin resistance and inflammation }\end{array}$ \\
\hline Perreault et al. (31) & $\begin{array}{l}\text { C18-ceramide levels increased in the skeletal muscle } \\
\text { cells isolated from individuals with type } 2 \text { diabetes }\end{array}$ \\
\hline \multicolumn{2}{|c|}{$\begin{array}{l}\text { Increased dihydroceramides/long chain ceramide with } \\
\text { fatty liver diseases }\end{array}$} \\
\hline $\begin{array}{l}\text { Apostolopoulou } \\
\text { et al. (25) }\end{array}$ & $\begin{array}{l}\text { Total serum dihydroceramides and hepatic } \\
\text { dihydroceramides (16:0, 22:0, and } 24: 1) \text { increased in } \\
\text { NASH } \\
\text { Hepatic dihydroceramides ( } 22: 0 \text { and } 24: 1) \text { increased } \\
\text { significantly in NASH and strongly associated with whole } \\
\text { body insulin-resistance }\end{array}$ \\
\hline $\begin{array}{l}\text { Wasilewska et al. } \\
\text { (26) }\end{array}$ & $\begin{array}{l}\text { Serum saturated ceramides species such as C14, C16, } \\
\text { C16:1, C18, and C18:1 significantly higher in children } \\
\text { with NAFLD }\end{array}$ \\
\hline Rosqvist et al. (27) & $\begin{array}{l}\text { Dietary saturated fat markedly induces the fatty liver } \\
\text { development, associated with increase serum total } \\
\text { ceramides specifically pronounced effect observed in } \\
\text { C16 ceramides } \\
\text { Dietary polyunsaturated fat prevents fatty liver } \\
\text { development associated with reduced serum } \\
\text { total ceramides }\end{array}$ \\
\hline
\end{tabular}

treating insulin resistance and fatty liver disease. More recent efforts have interrogated the value of targeting the CerS enzymes to alter the acyl-composition of ceramides to treat the comorbidities of obesity. These interventions are producing promising results.

\section{CerS1 and Muscle Insulin Resistance}

Skeletal muscle is the primary organ responsible for insulinmediated glucose uptake and utilization. During obesity, excess energy is delivered to muscle in the form of non-esterified fatty acids (NEFA) or lipoprotein-bound triglycerides. Excess delivery of these fatty acids to skeletal muscle causes insulin resistance, as the muscles adapt to the use of more plentiful energy source. This condition increases one's risk for type 2 diabetes and cardiovascular disease (33). Mechanistic studies have suggested that diacylglycerols (34) and ceramides (6) may contribute to skeletal muscle insulin resistance development.
CerS1 is the predominant isoform in muscle and the C18 ceramides that it produces are the major ceramide subspecies found in the tissue (9). Recent clinical observations identified higher levels of $\mathrm{C} 18$ ceramide in association with insulin resistance and inflammation in skeletal muscle (30). Another study found that $\mathrm{C} 18$-ceramide levels increased in skeletal muscle cells isolated from individuals with type 2 diabetes (31). To study the role of CerS1 in metabolic pathologies, researchers turned to rodent loss-of-function models (35). In these studies, high fat induced obesity led to an increase in C18 ceramides in muscle (35). Global ablation of CerS1 protected mice from high fat diet-induced weight gain. Moreover, the intervention increased energy expenditure, reduced adiposity, and improved insulin and glucose tolerance (35). Subsequently, the authors developed skeletal muscle-specific CerS1 knockout mice, demonstrating that tissue-specific ablation of the gene also improved glucose tolerance and insulin sensitivity (35). These observations suggest key roles for skeletal muscle $\mathrm{C} 18$ ceramides in the pathophysiology of obesity associated insulin resistance [Figure 2; (35)].

Turner et al. evaluated CerS1 as a potential drug target using novel small molecule inhibitors of the enzyme. The researchers developed P053, a specific inhibitor of CerS1 that displays nanomolar potency (36). PO53 displayed selectivity for CerS1 in mice by reducing $\mathrm{C} 18$ ceramide concentrations in skeletal muscle without changing other subspecies of ceramides (36). PO53treated animals showed improved skeletal muscle mitochondrial beta-oxidation relative to vehicle treated group (36). However, PO53 treated animals did not show any improvement in glucose tolerance and insulin sensitivity, despite having decreased intramuscular C18 ceramides (by 50\%) (36). This is in apparent contrast to the studies using genetic knockouts, which markedly altered glucose disposal. The contradictory observations could relate to differences in the diet induced obese mouse models used, the duration of the study or the degree of CerS1 inhibition that was achieved: (a) C57BL/6N mice were used in the knockout study, while the PO53 study used smaller C57BL/6J mice; (b) the knockout animals were fed for 12 weeks while the PO53 study were treated for only 4-6 weeks; and (c) the PO53 pharmacological treatment had a more modest effect on ceramides, reducing them by $\sim 50 \%$, compared to $>90 \%$ reduction in skeletal muscle of the knockout animals $(35,36)$. Therefore, further investigation of CerS1 inhibitors is warranted.

\section{CerS6 and NASH}

$\mathrm{NASH}$, characterized by hepatocellular lipid accumulation (steatosis) along with inflammation and varying degrees of fibrosis, is a serious condition affecting $1.5-6.45 \%$ of the population (1). Once afflicted, $\sim 10-15 \%$ of NASH patients progress to cirrhosis (37). The estimated annual economic burden of NASH in the USA is $\$ 103$ billion (38). NASH has no FDA-approved therapy, prompting many companies to race for approval of the first NASH-targeted drugs (38). Current disease management is primarily focused on promoting weight loss through lifestyle interventions, weight loss medication, and/or bariatric surgery (39). Limited prospective data are available on these options (39). 


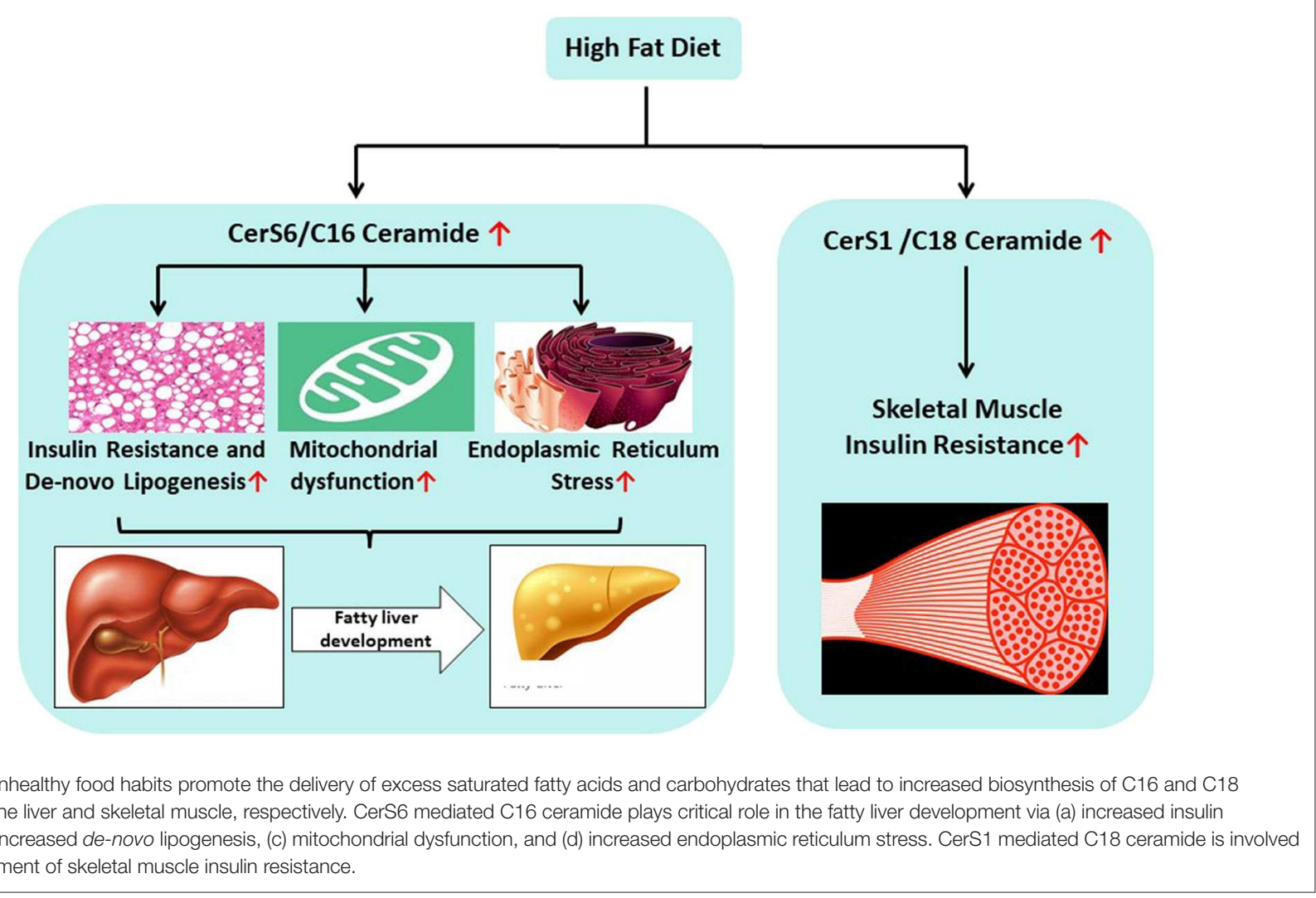

The pathology was originally interpreted to result from "dual-hits." The first hit, the steatosis that characterizes NAFLD, predisposes individuals to the second hits that include inflammation (40). More recently, complex "multiple-hit" hypotheses have been proposed, as numerous other factors including oxidative stress, mitochondrial dysfunction, and other insults are implicated in the pathology (41). Each stage is defined by specific risk factors and pathological mechanisms (42). As noted above, a number of studies indicate that sphingolipids such as ceramides, which can induce each of these elements, including inflammation, oxidative stress, and mitochondrial dysfunction, to drive NASH (13). Myriocin, a potent inhibitor of SPT, inhibits NASH progression in rodents $(43,44)$.

Several studies suggest that altering ceramide acylation patterns through CerS inhibition could also alter disease progression. Mice lacking both copies of the CerS2 gene display numerous liver abnormalities such as hepatocyte death and chronic apoptosis and regeneration $(45,46)$. The animals develop hepatoadenoma at 3-4 months of age, markedly reduced body weight, and hepatocarcinoma (around age 1 year) (45, 46). Furthermore, homozygous CerS2 knockout mice display decreased lipid accumulation and uptake in the liver (47). These animals displayed the expected reduction in very long chain acyl ceramides; however, they had a compensatory increases in C16ceramides $(45,46)$. This was interesting, as the C16-ceramides had been associated with hepatic insulin resistance (48) and disruption of the mitochondrial respiratory chain (49).
We subsequently studied heterozygous CerS2 knockout mice, which show a more modest pathology than their homozygous counterparts. The heterozygous CerS2 knockout mice show normal lifespan and no liver phenotype on normal chow diet (17) but phenotypes emerged when they were challenged with an obesogenic high fat diet (HFD). On the HFD, they displayed increased liver weight, triglycerides, macrophage infiltration, circulating liver enzymes, and plasma cholesterol, all of which are indicative of liver damage (17). These mice also displayed slightly impaired glucose tolerance, high fasting, and fed insulin levels, increased glucose mediated insulin secretion, reduced insulin sensitivity, decreased ambulatory activity, and increased fat to lean mass (17). As in the aforementioned study of the homozygous animals, the liver abnormalities were associated with reduced levels of very long chain ceramides coupled with the compensatory increase in C16-ceramides (17). In these studies, the increase in C16-ceramides could be explained by increased expression of CerS6. No changes were apparent on the normal chow diet. Mechanistic studies in primary hepatocytes obtained from the heterozygous CerS2 knockout mice revealed that the alteration in ceramide acylation attenuated insulin signaling and induced mitochondrial dysfunction (17). To recapitulate the compensatory increase of CerS6 expression, we overexpressed CerS6 in wild-type primary mouse hepatocytes. This intervention increased accumulation of C16 ceramides and compromised mitochondrial function, increased triglyceride accumulation and attenuated insulin signaling (17). 
Additional studies in mice have shown that diet induced NAFLD is associated with liver ceramide acylation patterns that paralleled the profile of the CerS2 knockouts. In general, high fat diets lead to increases in CerS6 expression and a concomitant elevation of C16-ceramides (50). Of note, in one study, overexpression of CerS2 was protective, presumably because it prevented the induction of CerS6 (50). In that study, CerS6 was shown to induce sterol regulatory element binding protein-1 (SREBP-1) cleavage and decrease levels of INSIG-1, leading to increased de-novo lipogenesis (DNL) (50). SREBP-1 is a master regulator of DNL in the liver and one of the primary insults that is dysregulated in the NASH pathophysiology (42). These authors reproduced our work (17), showing that CerS2 heterozygotes were susceptible to diet-induced steatohepatitis, exhibiting a pronounced endoplasmic reticulum (ER) stress response (50). The involvement of ER-stress was novel, but not surprising; Cinar et al. previously demonstrated that HFD-induced hepatic insulin resistance was associated with increased ER stress that was associated with elevated hepatic C16 and C18 ceramides (51).

Bruning et al. obtained the strongest evidence to date that CerS6 was pathogenic. They demonstrated that levels of CerS6 were increased in visceral and subcutaneous adipose tissue of obese humans, correlating positively with BMI and insulin resistance (18). These authors also found that the CerS6 product $\mathrm{C} 16$ ceramides were elevated in the visceral adipose of obese humans (18). They subsequently created CerS6 null mice, which allowed for a precise determination of the role of the enzyme in the development of obesity and fatty liver disease. The CerS6 null mice had the expected reduction in C16 ceramides. On a non-obesogenic chow diet, they had no obvious phenotype. However, the CerS6 null mice were protected from HFD-induced obesity (18). The change in body mass was completely explained by a reduction in adiposity, including a decrease in adipocyte size, serum leptin, adipose macrophage infiltration, and adipose pro-inflammatory gene expression (18). Additionally, the CerS6 null mice displayed improved insulin sensitivity and glucose tolerance (18). Turpin et al. also produced tissue-specific knockout mice, excising the gene from macrophages, brown adipocytes, and liver (18). Deletion of CerS6 from the brown adipose depots reduced diet induced obesity and improved mitochondrial beta oxidation, leading to elevations in energy expenditure (18). Liver-specific CerS6 null mice displayed a partial protection from diet induced obesity but a robust protection from glucose intolerance and insulin resistance explained by enhanced insulin signaling relative to wild type animals (18). Deletion of CerS6 from macrophages had no effect (18).

Brüning's group later identified a novel molecular mechanism through which CerS6 derived C16:0 ceramides alter mitochondrial dynamics, determining that the lipids interacted with mitochondrial fission factor (52). Deficiency of either CerS6 or mitochondrial fission factor encoded by the Mff gene protected mice from fatty acid-induced mitochondrial fragmentation in vitro. Moreover, the two proteins genetically interacted in vivo, participating in a linear pathway that accounted for obesity-induced mitochondrial fragmentation (52). Of note, an independent study reported that germline CerS6 knockout mice suffer neurobehavioral defects (53). This developmental defect was not observed by Brüning's group, which did not report any abnormal behavioral side effects of CerS6 deletion (18). These differences could be possibly explained by different knockout mouse generation strategies that were used $(18,53)$. However, from the safety pharmacology prospective it may be advisable to restrict compound use during pregnancy and/or to develop compounds that spares central nerves systems (CNS).

To develop therapeutic approaches to inhibit CerS6, we tested whether selective ablation of CerS6 using antisense oligonucleotides (ASO) was sufficient to reverse metabolic abnormalities in mice that were made obese by high fat diet (DIO mice) or leptin deficiency (ob/ob mice) (54). Delivery of the CerS6 ASO selectively reduced CerS6 expression by $90 \%$, predominantly in the liver. CerS6 knockdown reduced C16:0 ceramides by about 50\% in both liver and plasma (54). CerS6 ASO treatment efficiently lowered body weight gain and reduced body fat and fed (and fasted) blood glucose levels (1\% reduction in $\mathrm{HbA1c}$ ). Moreover, CerS6 inhibition improved oral glucose tolerance and insulin sensitivity (54). Both genetic knockout and ASO mediated genetic knock-down studies clearly demonstrated that inhibition of CerS6 activity ameliorated metabolic diseases including insulin resistance, type 2 diabetes and obesity [Figure $2 ;(17,18,54)]$. Therefore, further discovery of selective small molecule therapeutics targeting CerS6 inhibition is warranted.

\section{CerS5 and Obesity}

Gosejacob et al. developed CerS5 knockout mice and demonstrated that CerS5 null mice are viable and fertile and do not show any obvious morphological and phenotypic alterations on normal chow diet (55). However, when challenged with high fat diet CerS5 knockout animals protected against diet induced obesity and associated with reduced levels of leptin relative to wild type animals (55). Additionally, on diet induced obesity CerS5 knockout animals displayed improved glucose tolerance, insulin sensitivity, and reduced white adipose inflammation compared to wild type animals (55). This was also in contrast to the Brüning study, which showed no effect of CerS5 depletion (52).

\section{CONCLUSIONS}

Clinical observations and experimental studies in rodents suggest that specific ceramides have distinct roles in the pathophysiology of various human diseases. Studies reveal that altering sphingolipid acylation patterns impacts hepatic steatosis, adiposity, adipocyte size, adipokine secretion, macrophage infiltration, inflammation, insulin sensitivity, mitochondrial dysfunction, and ER stress. In particular, the ratio of long-chain ceramides (e.g., C16 and C18) to very long-chain ceramides (e.g., C24:0 or C24:1) appears to be a key factor in the development of metabolic disease. Therefore, inhibition of CerS6, and perhaps 
CerS1 and CerS5, may serve as an attractive therapeutic approach for treating insulin resistance, obesity, fatty liver, and NASH.

\section{REFERENCES}

1. Younossi ZM, Koenig AB, Abdelatif D, Fazel Y, Henry L, Wymer M. Global epidemiology of nonalcoholic fatty liver disease-meta-analytic assessment of prevalence, incidence, and outcomes. Hepatology. (2016) 64:7384. doi: 10.1002/hep.28431

2. Mittermayer F, Caveney E, De Oliveira C, Alexander Fleming G, Gourgiotis L, Puri M, et al. Addressing unmet medical needs in type 1 diabetes: a review of drugs under development. Curr Diabetes Rev. (2016) 13:30014. doi: 10.2174/1573399812666160413115655

3. Meikle PJ, Summers SA. Sphingolipids and phospholipids in insulin resistance and related metabolic disorders. Nat Rev Endocrinol. (2017) 13:7991. doi: 10.1038/nrendo.2016.169

4. Holland WL, Bikman BT, Wang LP, Yuguang G, Sargent KM, Bulchand S, et al. Lipid-induced insulin resistance mediated by the proinflammatory receptor TLR4 requires saturated fatty acid-induced ceramide biosynthesis in mice. $J$ Clin Invest. (2011) 121:1858-70. doi: 10.1172/JCI43378

5. Bikman BT, Summers SA. Ceramides as modulators of cellular and wholebody metabolism. J Clin Invest. (2011) 121:4222-30. doi: 10.1172/JCI 57144

6. Chavez JA, Summers SA. A ceramide-centric view of insulin resistance. Cell Metab. (2012) 15:585-94. doi: 10.1016/j.cmet.2012.04.002

7. Rosqvist F, Kullberg J, Ståhlman M, Cedernaes J, Heurling K, Johansson HE, et al. Overeating saturated fat promotes fatty liver and ceramides compared with polyunsaturated fat: a randomized trial. J Clin Endocrinol Metab. (2019) 104:6207-19. doi: 10.1210/jc.2019-00160

8. Chaurasia B, Summers SA. Ceramides - lipotoxic inducers of metabolic disorders. Trends Endocrinol Metab. (2015) 26:53850. doi: 10.1016/j.tem.2015.07.006

9. Park WJ, Park JW. The effect of altered sphingolipid acyl chain length on various disease models. Biol Chem. (2015) 396:693-705. doi: 10.1515/hsz-2014-0310

10. Merrill AH. Sphingolipid and glycosphingolipid metabolic pathways in the era of sphingolipidomics. Chem Rev. (2011) 111:6387-422. doi: 10.1021/cr2002917

11. Hannun YA, Obeid LM. Ceramide and the eukaryotic stress response. Biochem Soc Trans. (2000) 10:73-80. doi: 10.1016/s0962-8924(99)01694-3

12. Stratford S, Hoehn KL, Liu F, Summers SA. Regulation of insulin action by ceramide: Dual mechanisms linking ceramide accumulation to the inhibition of Akt/protein kinase B. J Biol Chem. (2004) 279:3660815. doi: 10.1074/jbc.M406499200

13. Summers SA, Chaurasia B, Holland WL. Metabolic messengers: ceramides. Nat Metab. (2019) 1:1051-58. doi: 10.1038/s42255-019-0134-8

14. Schmitz-Peiffer C. Targeting ceramide synthesis to reverse insulin resistance. Diabetes. (2010) 59:2351-53. doi: 10.2337/db10-0912

15. Ohta E, Ohira T, Matsue K, Ikeda Y, Fujii K, Ohwaki K, et al. Analysis of development of lesions in mice with serine palmitoyltransferase (SPT) deficiency - Sptlc2 conditional knockout mice. Exp Anim. (2009) 58:51524. doi: 10.1538/expanim.58.515

16. Hojjati MR, Li Z, Jiang XC. Serine palmitoyl-CoA transferase (SPT) deficiency and sphingolipid levels in mice. Biochim Biophys Acta Mol Cell Biol Lipids. (2005) 1737:44-51. doi: 10.1016/j.bbalip.2005.08.006

17. Raichur S, Wang ST, Chan PW, Li Y, Ching J, Chaurasia B, et al. CerS2 haploinsufficiency inhibits $\beta$-oxidation and confers susceptibility to dietinduced steatohepatitis and insulin resistance. Cell Metab. (2014) 20:68795. doi: 10.1016/j.cmet.2014.10.007

18. Turpin SM, Nicholls HT, Willmes DM, Mourier A, Brodesser S, Wunderlich $\mathrm{CM}$, et al. Obesity-induced CerS6-dependent C16:0 ceramide production promotes weight gain and glucose intolerance. Cell Metab. (2014) 20:67886. doi: 10.1016/j.cmet.2014.08.002

19. Boon J, Hoy AJ, Stark R, Brown RD, Meex RC, Henstridge DC, et al. Ceramides contained in LDL are elevated in type 2 diabetes and promote

\section{AUTHOR CONTRIBUTIONS}

SR researched the literature and wrote the manuscript. inflammation and skeletal muscle insulin resistance. Diabetes. (2013) 62:40110. doi: $10.2337 / \mathrm{db} 12-0686$

20. Neeland IJ, Singh S, McGuire DK, Vega GL, Roddy T, Reilly DF, et al. Relation of plasma ceramides to visceral adiposity, insulin resistance and the development of type 2 diabetes mellitus: the dallas heart study. Diabetologia. (2018) 61:2570-79. doi: 10.1007/s00125-018-4720-1

21. Chew WS, Torta F, Ji S, Choi H, Begum H, Sim X, et al. Large-scale lipidomics identifies associations between plasma sphingolipids and T2DM incidence. JCI Insight. (2019) 5:e126925. doi: 10.1172/jci.insight.126925

22. Hilvo M, Salonurmi T, Havulinna AS, Kauhanen D, Pedersen ER, Tell GS, et al. Ceramide stearic to palmitic acid ratio predicts incident diabetes. Diabetologia. (2018) 61:1424-34. doi: 10.1007/s00125-018-4590-6

23. Klein RL, Hammad SM, Baker NL, Hunt KJ, Al Gadban MM, Cleary PA, et al. Decreased plasma levels of select very long chain ceramide species are associated with the development of nephropathy in type 1 diabetes. Metabolism. (2014) 63:1287-95. doi: 10.1016/j.metabol.2014.07.001

24. Grammatikos G, Ferreiros N, Bon D, Schwalm S, Dietz J, Berkowski C, et al. Variations in serum sphingolipid levels associate with liver fibrosis progression and poor treatment outcome in hepatitis $\mathrm{C}$ virus but not hepatitis B virus infection. Hepatology. (2015) 61:812-22. doi: 10.1002/hep.27587

25. Apostolopoulou M, Gordillo R, Koliaki C, Gancheva S, Jelenik T, De Filippo $\mathrm{E}$, et al. Specific hepatic sphingolipids relate to insulin resistance, oxidative stress, and inflammation in nonalcoholic steato hepatitis. Diabetes Care. 41:1235-43. doi: 10.2337/dc17-1318

26. Wasilewska N, Bobrus-Chociej A, Harasim-Symbor E, Tarasów E, Wojtkowska M, Chabowski A, et al. Increased serum concentration of ceramides in obese children with nonalcoholic fatty liver disease. Lipids Health Dis. (2018) 17:216. doi: 10.1186/s12944-018-0855-9

27. Rosqvist F, Iggman D, Kullberg J, Cedernaes J, Johansson HE, Larsson A, et al. Overfeeding polyunsaturated and saturated fat causes distinct effects on liver and visceral fat accumulation in humans. Diabetes. (2014) 63:235668. doi: $10.2337 / \mathrm{db} 13-1622$

28. Huang H, Kasumov T, Gatmaitan P, Heneghan HM, Kashyap SR, Schauer $\mathrm{PR}$, et al. Gastric bypass surgery reduces plasma ceramide subspecies and improves insulin sensitivity in severely obese patients. Obesity. (2011) 19:2235-40. doi: 10.1038/oby.2011.107

29. Özer H, Aslan I, Oruç MT, Çöpelci Y, Afşar E, Kaya S, Aslan M. Early postoperative changes of sphingomyelins and ceramides after laparoscopic sleeve gastrectomy. Lipids Health Dis. (2018) 17:269. doi: 10.1186/s12944-018-0917-z

30. Bergman BC, Brozinick JT, Strauss A, Bacon S, Kerege A, Bui HH, et al. Muscle sphingolipids during rest and exercise: a C18:0 signature for insulin resistance in humans. Diabetologia. (2016) 59:785-98. doi: 10.1007/s00125-015-3850-y

31. Perreault L, Newsom SA, Strauss A, Kerege A, Kahn DE, Harrison KA, et al. Intracellular localization of diacylglycerols and sphingolipids influences insulin sensitivity and mitochondrial function in human skeletal muscle. JCI insight. (2018) 3:e96805. doi: 10.1172/jci.insight.96805

32. Montgomery MK, Brown SHJ, Lim XY, Fiveash CE, Osborne B, Bentley NL, et al. Regulation of glucose homeostasis and insulin action by ceramide acyl-chain length: a beneficial role for very long-chain sphingolipid species. Biochim Biophys Acta Mol Cell Biol Lipids. (2016) 1861:182839. doi: 10.1016/j.bbalip.2016.08.016

33. Meex RCR, Blaak EE, van Loon LJC. Lipotoxicity plays a key role in the development of both insulin resistance and muscle atrophy in patients with type 2 diabetes. Obes Rev. (2019) 20:1205-17. doi: 10.1111/obr.12862

34. Petersen MC, Shulman GI. Mechanisms of insulin action and insulin resistance. Physiol Rev. (2018) 98:21332223. doi: 10.1152/physrev.00063.2017

35. Turpin-Nolan SM, Hammerschmidt P, Chen W, Jais A, Timper K, Awazawa M, et al. CerS1-derived C18:0 ceramide in skeletal muscle promotes obesity-induced insulin resistance. Cell Rep. (2019) 26:110.e7. doi: 10.1016/j.celrep.2018.12.031 
36. Turner N, Lim XY, Toop HD, Osborne B, Brandon AE, Taylor EN, et al. A selective inhibitor of ceramide synthase 1 reveals a novel role in fat metabolism. Nat Commun. (2018) 9:3165. doi: 10.1038/s41467-018-05613-7

37. Rinella ME. Nonalcoholic fatty liver disease a systematic review. JAMA J Am Med Assoc. (2015) 313:2263-73. doi: 10.1001/jama.2015.5370

38. Younossi ZM, Blissett D, Blissett R, Henry L, Stepanova M, Younossi $\mathrm{Y}$, et al. The economic and clinical burden of nonalcoholic fatty liver disease in the United States and Europe. Hepatology. (2016) 64:157786. doi: 10.1002/hep. 28785

39. Chalasani N, Younossi Z, Lavine JE, Diehl AM, Brunt EM, Cusi K, et al. The diagnosis and management of non-alcoholic fatty liver disease: practice guideline by the American association for the study of liver diseases, American college of gastroenterology, and the American gastroenterological association. Hepatology. (2012) 55:2005-23. doi: 10.1002/hep.25762

40. Day CP, James OFW. Steatohepatitis: a tale of two "Hits"? Gastroenterology. (1998) 114:842-5. doi: 10.1016/S0016-5085(98)70599-2

41. Rosso N, Chavez-Tapia NC, Tiribelli C, Bellentani S. Translational approaches: from fatty liver to non-alcoholic steatohepatitis. World J Gastroenterol. (2014) 20:9038-49. doi: 10.3748/wjg.v20.i27.9038

42. Berlanga A, Guiu-Jurado E, Porras JA, Auguet T. Molecular pathways in non-alcoholic fatty liver disease. Clin Exp Gastroenterol. (2014) 7:22139. doi: 10.2147/CEG.S62831

43. Jiang M, Li C, Liu Q, Wang A, Lei M. Inhibiting ceramide synthesis attenuates hepatic steatosis and fibrosis in rats with non-alcoholic fatty liver disease. Front Endocrinol. (2019) 10:665. doi: 10.3389/fendo.2019. 00665

44. Yang RX, Pan Q, Liu XL, Zhou D, Xin FZ, Zhao ZH, et al. Therapeutic effect and autophagy regulation of myriocin in nonalcoholic steatohepatitis. Lipids Health Dis. (2019) 18:179. doi: 10.1186/s12944-019-1118-0

45. Pewzner-Jung Y, Brenner O, Braun S, Laviad EL, Ben-Dor S, Feldmesser E, et al. A critical role for ceramide synthase 2 in liver homeostasis II. Insights into molecular changes leading to hepatopathy. J Biol Chem. (2010) 285:10911-23. doi: 10.1074/jbc.M109.077610

46. Pewzner-Jung Y, Park H, Laviad EL, Silva LC, Lahiri S, Stiban J, et al. A critical role for ceramide synthase 2 in liver homeostasis: I. Alterations in lipid metabolic pathways. J Biol Chem. (2010) 285:1090210. doi: 10.1074/jbc.M109.077594

47. Park WJ, Park JW, Merrill AH, Storch J, Pewzner-Jung Y, Futerman AH. Hepatic fatty acid uptake is regulated by the sphingolipid acyl chain length. Biochim Biophys Acta Mol Cell Biol Lipids. (2014) 1841:175466. doi: 10.1016/j.bbalip.2014.09.009
48. Park JW, Park WJ, Kuperman Y, Boura-Halfon S, Pewzner-Jung Y, Futerman $\mathrm{AH}$. Ablation of very long acyl chain sphingolipids causes hepatic insulin resistance in mice due to altered detergent-resistant membranes. Hepatology. (2013) 57:525-32. doi: 10.1002/hep.26015

49. Zigdon H, Kogot-Levin A, Park JW, Goldschmidt R, Kelly S, Merrill AH, et al. Ablation of ceramide synthase 2 causes chronic oxidative stress due to disruption of the mitochondrial respiratory chain. J Biol Chem. (2013) 288:4947-56. doi: 10.1074/jbc.M112.402719

50. Kim YR, Lee EJ, Shin KO, Kim MH, Pewzner-Jung Y, Lee YM, et al. Hepatic triglyceride accumulation via endoplasmic reticulum stress-induced SREBP-1 activation is regulated by ceramide synthases. Exp Mol Med. (2019) 51:116. doi: 10.1038/s12276-019-0340-1

51. Cinar R, Godlewski G, Liu J, Tam J, Jourdan T, Mukhopadhyay B, et al. Hepatic cannabinoid-1 receptors mediate diet-induced insulin resistance by increasing de novo synthesis of long-chain ceramides. Hepatology. (2014) 59:143-53. doi: 10.1002/hep.26606

52. Hammerschmidt P, Ostkotte D, Nolte H, Gerl MJ, Jais A, Brunner $\mathrm{HL}$, et al. CerS6-derived sphingolipids interact with $\mathrm{mff}$ and promote mitochondrial fragmentation in obesity. Cell. (2019) 177:1536-52.e23. doi: 10.1016/j.cell.2019.05.008

53. Ebel P, Vom Dorp K, Petrasch-Parwez E, Zlomuzica A, Kinugawa K, Mariani $\mathrm{J}$, et al. Inactivation of ceramide synthase 6 in mice results in an altered sphingolipid metabolism and behavioral abnormalities. J Biol Chem. (2013) 288:21433-47. doi: 10.1074/jbc.M113.479907

54. Raichur S, Brunner B, Bielohuby M, Hansen G, Pfenninger A, Wang B, et al. The role of C16:0 ceramide in the development of obesity and type 2 diabetes: CerS6 inhibition as a novel therapeutic approach. Mol Metab. (2019) 21:36-50. doi: 10.1016/j.molmet.2018.12.008

55. Gosejacob D, Jäger PS, Dorp K Vom, Frejno M, Carstensen AC, Köhnke M, et al. Ceramide synthase 5 is essential to maintain C16:0-ceramide pools and contributes to the development of diet-induced obesity. J Biol Chem. (2016) 291:6989-7003. doi: 10.1074/jbc.M115.691212

Conflict of Interest: SR is an employee of Evotec International GmbH, Germany.

Copyright (C) 2020 Raichur. This is an open-access article distributed under the terms of the Creative Commons Attribution License (CC BY). The use, distribution or reproduction in other forums is permitted, provided the original author(s) and the copyright owner(s) are credited and that the original publication in this journal is cited, in accordance with accepted academic practice. No use, distribution or reproduction is permitted which does not comply with these terms. 